\title{
Baluartes contra tenazas El caso de la Goleta en 1565
}

\section{José Javier de Castro Fernández ${ }^{\text {, Javier Mateo de Castrob }}$}

Asociación Española Amigos de los Castillos. Valladolid. España. ajjdecastro@yahoo.es, bjmat87@gmail.com

\begin{abstract}
One of the most interesting debates around the fortification systems will take place in Tunisia's stronghold, after the Turkish siege of Malta in 1565. The court backed Francesco Paciotto's design, also supported by King Philip II, that consisted of defending the front through a pointed-bulkwards system. This project would be supported by the majority of the engineers in the kingdom. However, a servicemen engineer opposed this thesis, Alonso de Pimentel. He will propose resuming the pincers system that Emperor Charles V liked so much. The idea was to build two big bulkwards linked by a straight wall, a model that had obtained a good result during the defense of Malta, and which had also been used in Milan by Alonso de Pimentel. Finally King Felipe II's proposal was imposed, due to his enthusiasm of the Italian treatises, which implemented the triangular model in La Goleta. The conquest of the fortress in 1574 will show the mistake.
\end{abstract}

Keywords: Alonso Pimentel, Franceso Paciotto, Bulkward, Tunisia.

\section{Introducción}

En 1565, tras el asedio turco a Malta, en la Goleta de Túnez tendrá lugar uno de los más interesantes debates entorno a la construcción de la nueva fortaleza. La Corte propone el diseño de Francesco Paciotto, secundado por Felipe II y su Consejo de Guerra, que consiste en defender el frente de ataque mediante un sistema triangular de tres baluartes en punta. Este proyecto también contará con el apoyo de la mayoría de ingenieros que participan en los diseños de la nueva fortificación de Malta y de los virreinatos de Sicilia y Nápoles. Sin embargo, existe un militar-ingeniero, Alonso de Pimentel, que se opone, siendo más partidario de retomar los postulados del sistema atenazado que tanto le gustaba a Carlos V y a alguno de sus principales colaboradores: Ferrante Gonzaga, Luis Pizaño, Pedro Luis Escrivá, o Pedro Prado. La idea es construir dos grandes baluartes unidos por una cortina recta, sistema que había tenido buen resultado en la defensa de Malta (fuertes de
San Telmo y San Miguel). Este mismo modelo atenazado ya lo había empleado Alonso Pimentel en la ciudadela de Milán. Sin embargo, se impone la tesis de Felipe II, verdadero entusiasta de la tratadística italiana representada por De Marchi, y se construye la defensa triangular, no solo en la Goleta de Túnez sino que también se modifica la ciudadela de Milán con el mismo sistema de tres baluartes en punta. El asedio a la Goleta de 1574 puso a cada sistema de defensa en su lugar y el proyecto no realizado de Alonso Pimentel fue elogiado años más tarde por Carlos Theti en su tratado y declamado por el poeta Alonso de Salamanca.

\section{Fortificaciones provisionales}

A comienzos de 1565 llegan noticias alarmantes a la Corte española sobre la formación de una gran flota turca para el verano de ese año pero desconociendo su objetivo. Dos lugares son los 
que cuentan con mayores probabilidades, Malta y La Goleta de Túnez. El informe remitido por García de Toledo a tenor del segundo enclave en abril de 1565 es claramente desolador y muestra las dificultades de poder resistir con éxito un asedio: "Los fosos son bajos de caída y de poco hondo por la parte de las dos baterías; las espaldas de los caballeros son muy débiles y muy desconvenientes a la batería que allí se puede esperar que habrá; la plaza de dentro muy pequeña y muy aparejada por la poca altura a subjetarla de fuera con dos caballeros". (AGS.E1129). Ante esta tesitura desde la Corte se idea una fortificación provisional para la Goleta enviando al ingeniero Luis Escrivá para que lo ejecute. Este proyecto de urgencia consiste básicamente en "alargalla creciendo los baluartes y sacándolos mas”, además de construir un caballero en el interior de la fortaleza. El objetivo de esta reforma es claro, los baluartes, diseñados en los años 40 del siglo XVI han quedado desfasados, entre otras cosas por su tamaño, por lo que se persigue es ampliar tanto las caras como los flancos.

Este proyecto provisional será duramente criticado por el nuevo gobernador de la plaza Alonso Pimentel quien en junio de 1565 informa al rey: “En lo de la fortificación ... quedará tiempo de hazello a boca y en quedar mi parecer como hombre que he visto lo que conviene que es bien diferente de lo que se puede juzgar de lexos y así no se pasará adelante en el designio que V.Mg. me imbio ni en ningún otro hasta que yo diga a V.Mg. lo que mas conveniente me parece" (AGS.E-486). Por tanto, Alonso Pimentel paraliza las obras provisionales que estaba ejecutando el ingeniero Luis Escrivá y adelanta que él tiene redactado un nuevo proyecto que, como veremos más adelante, se asemejaba al que trazó en 1562 para la ciudadela de Milán.

No será el único que critique estas obras por cuanto Sancho de Leiva, con un tono mucho menos diplomático, afirma con rotundidad: "no me ha parecido bien, porque al fin es remiendo, y por remiendo quedara como los otros que se han hecho allí de 30 años a esta parte”. Leiva demuestra ser un gran conocedor de las vicisitudes de la Goleta desde el error de planteamiento de
1535 con la construcción de una fortaleza triangular, la dificultad para transformarla posteriormente en una de planta rectangular, y que cuando se terminó sus medidas eran ya totalmente insuficientes para una fortificación de la segunda mitad del siglo XVI.

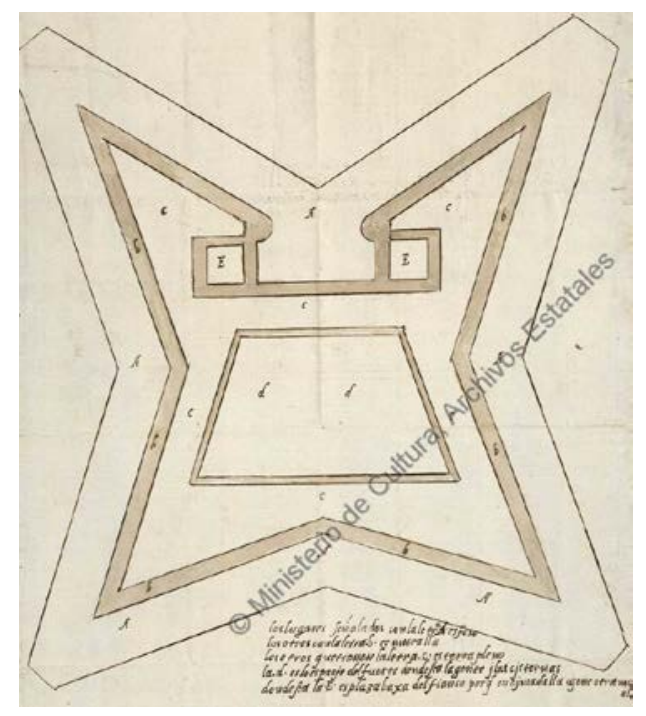

Fig. 1- Planta de San Telmo de Malta por Pedro Prado. 1552. AGS.MPD-18-152.

Los tres ingenieros militares Alonso Pimentel, García de Toledo y Sancho de Leiva que se encuentran en la zona de Malta-Sicilia-Túnez proponen nuevas obras provisionales para la Goleta. El objetivo es claro, pues como afirma Alonso Pimentel que "siendo tan chica esta plaza ... no puede tener ningún reparo en ella” por lo que propone salir fuera y construir diversas defensas exteriores: "no siendo la Goleta plaza fuerte, que no quiere él perderse dentro della sino defendella de fuera, y ansi la ha cercado de ciertos reparos de tierra". También Toledo es partidario de realizar obras exteriores y propone un gran baluarte de tierra en la zona del estaño o lago. Por su parte Sancho de Leiva añade la excavación de un foso exterior porque: "se harán los fosos que es la mayor y mejor fuerza que puede tener la Goleta; los cuales yo haría tan anchos y tan grandes que toda la fuerza quedase isla" (AGS.E1129). Como vemos, los tres ingenieros-militares están de acuerdo en que la defensa de la Goleta debe hacerse desde obras provisionales situadas 
delante de la Goleta. Todas estas propuestas sacan de quicio al pobre ingeniero Escrivá, quien en agosto de ese año informa al rey: "son reparos muy inútiles y costosos", llegando a afirmar de una forma un tanto paranoica "que si pongo inconveniente alguno en lo que el (Alonso Pimentel) traza me cortara la cabeza".

\section{El proyecto de Paciotto}

Pese a las buenas intenciones de los tres ingenieros militares el monarca Felipe II tenía ya definido como iba a ser la obra definitiva para la Goleta, proyecto que fue gestado por el ingeniero Paciotto en su visita a la Corte en 1561. Este ingeniero pasará a ser el favorito del monarca al que le encarga la revisión de las fortalezas del Milanesano, sobre todo la nueva fortificación que había construido Alonso Pimentel en 1562 en la fortaleza de los Sforza, y que diseñará las nuevas ciudadelas pentagonales de Amberes y Turín.

Paciotto es un buen representante del periodo llamado "el optimismo de la traza italiana" (Cobos, 2014) del que Felipe II será un entusiasta seguidor ya que considera que con este modelo de fortificación basado en la tratadística la cuestión está resuelta. Es una solución burócrata que delata fallos en su formación castrense, por el contrario su padre, gran militar, conocía perfectamente la problemática de la fortificación y que no existe una varita mágica que lo solucione, por ello era un gran partidario del sistema atenazado, un modelo antagónico del clásico sistema italiano o de tratados.

El propio Paciotto en su "Diario" escribe "Pacioto fece il disegno de la Goletta qual su poi alterato da don Aloisio Pimentello" (Marías, 2001), añadiendo con cierto sarcasmo: "che dio voglia che sia cosa buona", en clara alusión al sexto baluarte que añadirá, como luego veremos, Alonso Pimentel en 1569 situado hacia el estaño. No deja de ser curioso que Paciotto no hiciera ninguna mención posterior en su diario al fracaso de su diseño tras el asedio turco a la Goleta de 1574.

El proyecto de Paciotto para La Goleta consiste en una fortificación de planta regular con cinco baluartes, tres situados en el frente de ataque de Cartago y dos situados hacia Radiz. En síntesis no deja de ser el proyecto de una ciudadela como las que está diseñando para Turín o Amberes, con tres baluartes hacia el frente de ataque y dos hacia el interior de la ciudad. Pero con un importante error de planteamiento por cuanto las ciudadelas que diseña se sitúan en un vértice de la población, generando un frente de ataque doble, mientras que en el caso de La Goleta sólo hay un frente de ataque y éste es recto.

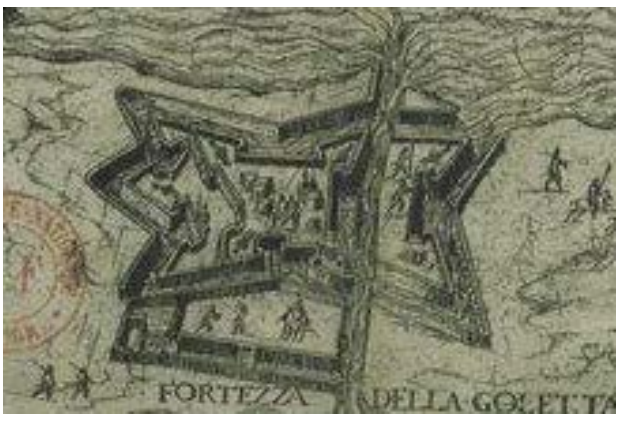

Fig. 2-Planta de la Goleta según el proyecto de Paciotto. (está invertido)

Esta diferenciación es básica para el sistema de defensa del flanco de los baluartes al quedar el frente recto como si estuviera defendido por una fortaleza triangular, planta que en todos los tratados de fortificación siempre se ha considerado la más imperfecta. Además comete un segundo error al unir la nueva fortificación de tres baluartes a la antigua fortaleza formando un todo, lo que determinaba que cuando fuera tomado la parte exterior también caería el recinto interior o antiguo.

Los ingenieros militares, con Alonso Pimentel a la cabeza, son concientes de este error de planteamiento y proponen un modelo distinto al de Paciotto, precisamente el que hizo Alonso Pimentel en la ciudadela de Milán. Consistía, en el frente de ataque, en una tenaza con dos baluartes y una cortina recta. Pero Felipe II en octubre de 1565 contesta: "Habiéndose tomado resolución que en la Goleta de Túnez se haga la fortificacion que ha parescido convenir ..., y mandado que el Fratin nuestro ingeniero vaya a ponerla en ejecución”. 
Cuando Fratín llega a la Goleta, viene con la lección bien aprendida de que debe ejecutar escrupulosamente el proyecto de Paccioto-Felipe II y por ello no se le dan poderes de ingeniero sino solo de veedor. La misiva de diciembre de ese año es muy clara: "porque vais a entender en la fortificacion que se ha de hacer en la Goleta de Tunez... sirviendo de veedor" (AGS.CG-28), lo que a la postre significa controlar la ejecución de la obra pero sin poder para variar el proyecto.

\section{La junta de ingenieros de $\mathbf{1 5 6 6}$}

Ante el proyecto que viene impuesto desde la Corte, el virrey de Sicilia, García de Toledo, trata de modificar el criterio real y a primeros de 1566 forma una gran junta de ingenieros reuniendo a los destinados en el virreinato de Nápoles, Sicilia y los que estaban en Malta y en la Goleta. La relación que confecciona García de Toledo es impresionante, con trece de los principales ingenieros de la Corona Hispánica: Ascanio de la Corna, Chapin Viteli, Gabrio Cervello, Julián Romero, el Fratin, Alonso Pimentel, Vespasiano Gonzaga, Juan Tomas Escala, Jacobo Lantieri, Antonio Conde, Sancho de Leiva, Pedro de Padilla y el propio virrey García de Toledo. Si bien en la reunión que se celebra en la Goleta en abril de 1566 faltan Viteli, Gonzaga, Lantieri y Conde.

García de Toledo, como buen político, debía guardar las formas y no podía decir abiertamente a Felipe II que el objetivo de la junta era desprestigiar el proyecto de Paciotto y por ello informa que van a tratar de ciertas obras provisionales en el exterior de la fortaleza. $\mathrm{Si}$ bien, no todos eran tan diplomáticos y Sancho de Leiva escribía al rey en agosto de 1566 estas durísimas palabras: "Yo solo dire una cosa, que no he visto a nadie que haya visto aquella traza de los que la entienden que les contente. Yo soy de la mesma opinión. Por lo cual suplico a V.M. lo mande tornar a ver antes que se haga cosa que después si descontenta sea malo de remediar".

Alonso Pimentel realiza diversas propuestas que son asumidas por la Junta de Ingenieros. En concreto considera que es necesario excavar "dos trincheras una a la parte de Radiz y otra a la de Cartago, detrás de dos fosos de agua que tiene hechos en entrambas partes lexos de la muralla de la Goleta por cada frente de 450 o 500 pasos ... siendo estas trincheas favorescidas de la fortaleza". Su idea es similar a la de Sancho de Leiva cuando propuso convertir la Goleta en una isla haciendo dos fosos que comunicaran el mar con el estaño. Ahora se perfila más la idea y se considera que estos fosos deben tener un gran tamaño -tanto en anchura como profundidad- y debieran ubicarse al amparo de las armas de fuego de la fortaleza pero lo suficientemente lejos de ésta para que en caso de asedio cuando "plantasen artilleria fuese tan lejos que no hiciese el efecto que habia de hacer"

(AGS.E-1395)

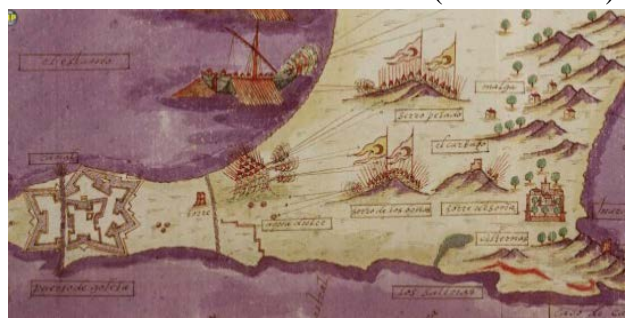

Fig. 3-La Goleta y fortificaciones exteriores. Archivo de Estado de Turín.

Dos son las razones por las que Alonso Pimentel coloca esta tenaza tan separada de la Goleta Vieja. La primera es alejar lo más posible la artillería turca de la fortaleza para que su potencia de tiro sea lo más ineficaz posible; y, la segunda, es que debe colocar todo el nuevo sistema: un foso pegado a la fortaleza vieja, la tenaza con sus baluartes, un camino cubierto y el gran foso exterior. Mientras que Toledo no consideraba necesaria tanta distancia y sitúa las nuevas defensas casi pegadas a la Goleta Vieja (como hará Fratín siguiendo el proyecto de Paciotto), lo que fue duramente criticado por Sancho de Leiva cuando afirma que se ha construido: "en derecho del baluarte que mira a Tunez un espontón que sale muchas canas y es tan ancho que iguala con el dicho baluarte".

Tenemos por tanto a los ingenieros-militares tratando de impedir la construcción de un frente triangular y de imponer el sistema atenazado, precisamente el que había salvado Malta del asedio turco de 1565. El propio García de 
Toledo se lo recordaba con bastante sorna en enero de 1566 al gran maestre de Orden de San Juan de la Valetta. "(querrán) SS ${ }^{a}$ Ilma, y aquellos señores acordar del grande util que recibieron el verano pasado de Sant Elmo con ser una fuerza tan pequeña y mal entendida, pues alli no solamente perdieron el animo y la mejor gente que tenían". Haciendo un claro elogio del sistema basado en la tenaza del fuerte de San Telmo que había construido el ingeniero Pedro Prado en 1552 y que recordemos él mismo defendía ahora para la nueva fortificación de la Goleta al proponer que "se podria hacer una cortina con dos caballeros que la guardasen por los lados, y asi no podria ser este sitio combatido, las espaldas guardada la misma Goleta, ni pueden los enemigos quitar que no se socorran los unos a los otros".

Este sistema defensivo -dos baluartes y una cortina recta- no era muy usado en Italia, y por tanto los ingenieros italianos, como el propio Vespasiano Gonzaga afirmaba "que en plazas situadas en campo llano y raso no se hacen estas tenazas que en Italia se dicen a cola de Milano". Aunque curiosamente algunos de los principales aliados de la Corona Hispánica en Italia como Cosme de Médici, Manuel Filiberto de Saboya o el papa Pablo III de Farnesio sí llegaron a construir diversos fuertes con tenazas y tijeras como Forte Falcone, Forte Stella, Mondevi y la Roca Pauliana de Perugia; o lo que es todavía más curioso los turcos-argelinos también construyeron fuertes atenazados como Bizerta o Argel, y mientras Felipe II que disponía de los técnicos y de una más que demostrada experiencia de éxito con este sistema, lo rechaza de plano.

\section{La ciudadela de Milán}

En 1560 comienza la construcción de una nueva fortificación para el castillo sforzesco de Milán. El gobernador duque de Sesa encarga su planificación a Alonso Pimentel. Hasta la fecha este personaje no pasa de ser tratado como cierto militar por Fernando Marías o un perfecto desconocido para Carlos Promis, al que se refiere como Luis Pimentel, o Jalel Akacha que incluso dice que hay dos hermanos, Alonso y
Luis. Sin embargo durante las guerras de Italia 1557- alcanzó el grado de maestre de campo general y es citado también como diseñador de cañones en el tratado de Luis Collado. Lo más interesante de la propuesta de Alonso Pimentel para el castillo de Milán es la defensa del frente de ataque encabalgando sobre la muralla de la ciudad una gran tenaza con dos grandes baluartes unidos por una cortina recta. Este proyecto, que se empieza a ejecutar, se modificará precisamente por los mismos personajes que nos encontramos en la Goleta de Túnez: Escrivá, Pacciotto y Fratín. Lo que proponen es lo mismo que trazan para la Goleta, defender un frente recto con tres baluartes en triángulo. Es magnífico el plano de Corbeta de 1567 donde se puede apreciar perfectamente lo que proponen estos ingenieros con un gran baluarte central con sus flancos completamente expuestos a la artillería enemiga.

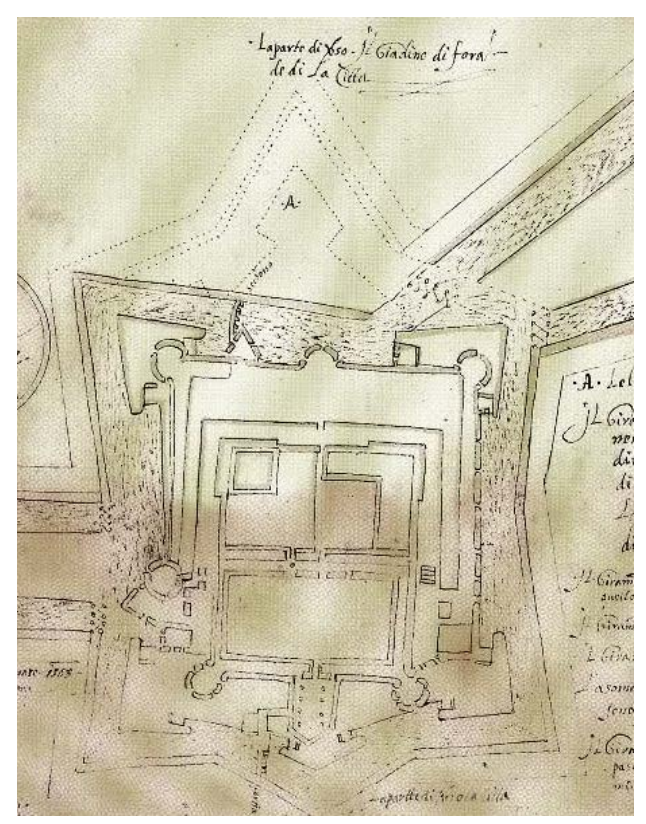

Fig. 4-Planta del castillo de Milán por Tomaso Corbeta, 1567, donde traza un gran frente triangular con un tercer baluarte central.

\section{El proyecto de Alonso Pimentel}

El proyecto de Alonso Pimentel para la Goleta lo conocemos gracias a un texto -cantos en octava 
rima- que compuso el militar Alonso Salamanca durante su cautiverio en poder de los turcos, quien había servido en la Goleta durante más de 25 años y por tanto fue testigo de excepción de todo lo que aconteció durante la construcción de la ampliación de la fortaleza (González, 1992).
“También diçen si lo que allí traçado por Pimentel en partes que aquí alego de Sant Pedro y Elifonso fuera obrado, sin duda oviera sido nuestro el juego, porque cassi çien baras más sacado cada mura al Estaño, gran sosiego fuera y más formada la defenssa de aquella fortaleza tan inmensa

También si Sant Martín fuera sacado de punta hasta la mar, como él dezia, el defenderse fuera más formado, mas el ingeniero dixo no podía passar de gran consejo lo mandado. Que çierto si anssi aquesto se haçía nunca nuestro enemigo lo tomara ni su bandera en él jamás plantara.

También diçen allí, fue mal dejado San Pedro y Sant Martín juntos en uno $y$ los fosos vaxos que han cegado, que fue conçejo sin provecho alguno, que haviéndole el enemigo en nuevo entrado pudiera de lo viejo estar ayuno y gastar más pólvoras y pertrechos que no en tomar lo nuevo y otros hechos"

Alonso de Salamanca niega tajantemente que la autoría fuera de Fratín, sino que el proyecto vino determinado desde la corte de Madrid, al afirmar: "mas el ingeniero dixo no podia passar de gran consejo lo mandado". Los datos que aporta sobre el proyecto de Alonso Pimentel consisten que en el frente de ataque, por donde los turcos entraron en la Goleta en el asedio de 1574, los baluartes de San Pedro y San Martín, tenían que haberse construido al borde mismo de la laguna y del mar (de la punta hasta la mar); y además elimina el baluarte central de San Felipe (juntos en uno). Con estos mimbres, la planta es clara: coloca en el frente de ataque una gran tenaza exterior con dos baluartes situados en los extremos, apoyados por las defensas naturales del mar y la laguna, y unidos por una gran muralla recta. Da también otro dato interesante, y que coincide con lo que antes hemos explicado: que esta obra debía estar separada de la fortaleza vieja (pudiera de lo viejo estar ayuno), y así evitar que cuando los turcos tomaran los baluartes exteriores los defensores pudieran mantenerse en la Goleta Vieja.

Este proyecto de Alonso Pimentel fue copiado íntegramente por el ingeniero Carlo Theti en su tratado "Discorsi delle fortificationi, espugnationi, \& difese delle città, \& d'altri luoghi”, si bien con la ligera diferencia de que mientras Pimentel firmó su proyecto antes del asedio de 1574, Theti lo escribió después del asedio, cuando se vio claramente los defectos y virtudes de lo realizado y lo proyectado.

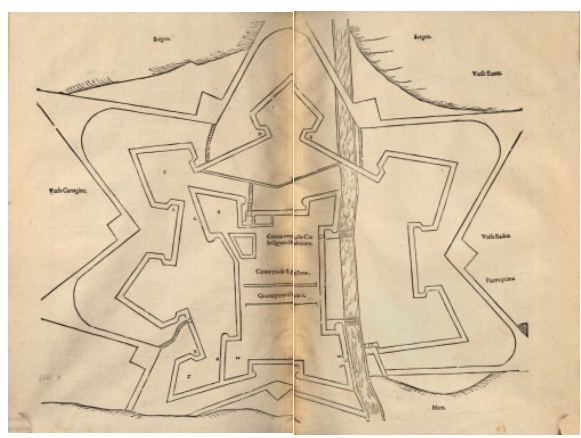

Fig. 5-Planta de La Goleta en el tratado de Carlos Theti. (está invertido)

Carlos Theti en su tratado presenta de forma muy didáctica en dos planos las diferencias entre ambos diseños. En el primero muestra lo construido por Paciotto y en el segundo muestra una gran tenaza, separada de la Goleta vieja, con dos grandes baluartes situados al borde del mar y del lago, y que disponen de amplias cañoneras en los flancos, añade un gran foso delantero con su camino cubierto. En su tratado Theti critica, entre otras cuestiones, que los baluartes de la Goleta Vieja y Nueva estén juntos; que se debió excavar un foso entre ambas fortalezas; que las troneras de los flancos de los nuevos baluartes son fácilmente embocados por el enemigo; o que dos baluartes están muy juntos.

Como podemos apreciar, tanto las críticas que hace Theti al proyecto de Paciotto como la solución que aporta son exactamente las mismas que defendió inútilmente Alonso Pimentel ante Felipe II en 1565. Si bien Theti añade otra cuestión al incidir que fue un error diseñar la Goleta para defenderse con mosquetes, aunque 
no debemos olvidar que una de las máximas de la fortificación de la Corona Hispánica es que la distancia máxima de la línea de defensa debe ser el alcance de un mosquete y no de un cañón. Sin embargo Alonso Pimentel tenía la solución a este dilema porque pensaba emplear la carabina árabe que tiene gran alcance y precisión, con lo que lograba cubrir la distancia de la línea de defensa.

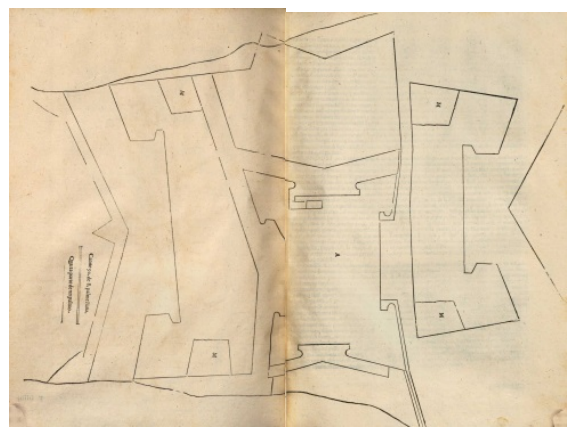

Fig. 6-Proyecto de Carlos Theti para la Goleta que coincide con lo proyectado por Alonso Pimentel en 1565. (está invertido)

Alonso Pimentel tenía una importante formación como militar y como ingeniero. Su maestro debió ser Pedro Prato, ingeniero que trabajó a las órdenes de Pedro Luis Escrivá en San Telmo de Nápoles y que en 1552 construirá dos de las principales fortificaciones atenazadas de la Corona Hispánica: San Telmo y San Miguel de Malta, obras que fueron avaladas por Juan de Homedes, Gran Maestre de la Orden de San Juan, que orgullosamente lo reflejó en su epitafio. También participó como ingeniero en la famosa conquista de la plaza norteafricana de Mahdia y allí coincidió precisamente con Alonso Pimentel, Sancho de Leiva, García de Toledo y curiosamente también con Carlos Theti, todos ellos grandes defensores del sistema atenazado.

Alonso Pimentel tenía muy clara la importancia del través al igual que Pedro Luis Escriva quien en su tratado escribe que "las cañoneras -quanto mas cubiertas están y menos descubren por costado mejores son”, y así lo demostró en el asedio de Cendal cuando "los enemigos no podian pelear ni se osavan asomar a tirar como solian al parapeto del cavallero por amor de las piezas que de fianco jugaban que don Alonso
Pimentel aiva plantado". También era un especialista en la fabricación de cañones. El propio Luis Collado lo cita en su tratado "Platica manual de artillería”, añadiendo que era un gran aficionado a los basiliscos, arma de asedio favorita de los turcos, y que fueron una de las principales causas de la rápida destrucción de las murallas de la nueva Goleta, por ello no debemos olvidar cuando Alonso Pimentel proponía defensas exteriores para que cuando los turcos "plantasen artilleria fuese tan lejos que no hiciese el efecto que habia de hacer".

\section{El sexto baluarte de la Goleta}

La obra debe comenzar en 1569 cuando el Fratín regresa a España, medio que permite a Alonso Pimentel tener la suficiente libertad para diseñar $\mathrm{y}$ modificar la traza original construyendo un sexto baluarte. Su principal objetivo es tratar de defender el sector del lago que no cuenta con la debida protección, sobre todo cuando tras la conquista de los turcos-argelinos de Ochali de la ciudad de Túnez, éste planea un ataque con barcos por el lago y desembarcar en este punto. Su atribución a Alonso Pimentel es clara, primero por la denominación del baluarte: San Ildefonso que es igual que San Alfonso o San Alonso, y segundo que en un informe de 1566 sobre la evolución de la fortificación no se menciona este sexto baluarte, y tampoco consta en un dibujo que relata el ataque turco de 1570 y existe un plano de Lafrey que dibuja el fuerte de cinco baluartes y además añade el proyecto del sexto baluarte.

Su construcción debió ser bastante rápida, por cuanto en una relación del estado de las obras de abril de 1570 se informa que el baluarte de San Ildefonso "está hasta el cordón", sin olvidar que el propio Paciotto en su Diario nos menciona que Alonso Pimentel modificó su proyecto original.

\section{Conclusiones}

Tras la conquista de la Goleta en 1574, el monarca Felipe II reúne Junta de Ingenieros en Madrid y lo que rechazó en la reunión de la Goleta de 1566 debe aceptarlo ahora, lo que originará una verdadera eclosión del empleo de 
la tenaza para la defensa de los puntos más comprometidos de una fortaleza o recinto amurallado. A título de ejemplo, solo en la zona del Mediterráneo, tenemos como se paraliza el proyecto para Taranto de Benvenuto, abandonándose la construcción típica triangular de tres baluartes y se adopta el nuevo proyecto de Atendolo con la construcción de dos grandes baluartes unidos por una cortina recta.

O la tenaza de Campi para Siracusa, de Tiburcio Spanochi para Milazzo, Vespasiano Gonzaga para Benidorm y Peñíscola, o las que se proponen para Medina en Malta o para el castillo de San Salvador de Mesina donde el virrey resume la obra a ejecutar con un lacónico "se ha de hacer solamente una cortina y dos baluartes muy gallardos en la frente de tierra" (De Castro, 2012)

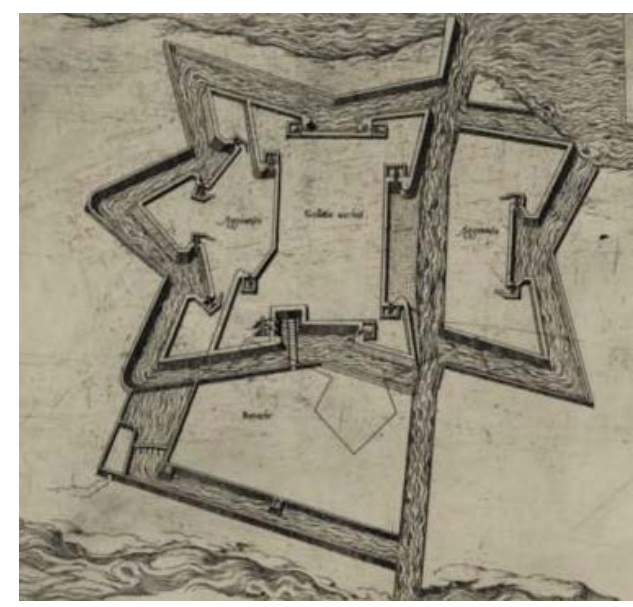

Fig. 7-Atlas de Lafrey donde se traza el sexto baluarte ejecutado por Alonso Pimentel. 1569.

\section{Referencias}

Akacha J., Garulli M. (1994). “Architetti e ingegneri militari italiani al presidio della Goletta di Tunisi (1535-1574). En Architetti e ingegneri militari italiani all'estero dal XV al XVIII secolo. Castella 44.

Cobos F., Cámara A., coord. Revuelta B. coord. (2014). "Pedro Luis Escrivá y el primer tratado de fortificación moderna. Nápoles 1538”. En Ingenieros del Renacimiento. Madrid.

Cobos F., De Castro J.J., Sánchez Gijón A. (2000). Luis Escrivá, su Apología y la fortificación imperial. Valencia.

De Castro J.J., Cobos F. (2000). "El debate en las fortificaciones del Imperio y la monarquía española 1535-1574”. En Las Fortificaciones de Carlos V. Madrid.

De Castro J.J., Cuadrado A. (2012). "Las fortificaciones de la Corona Hispánica en el Mediterráneo durante los siglos XVI-XVII (1492-1700)”. En Actas IV Congreso de Castellología. Asociación Española Amigos de los Castillos. Madrid.

www.castillosasociacion.es/sites/castillosdeespana.es/files/pdf/pon5.pdf

González R. (1992). "La pérdida de La Goleta y Túnez en 1574, y otros sucesos de historia otomana, narrados por un testigo presencial: Alonso de Salamanca”. Anaquel de Estudios Árabes, III, 1992.

Maggiorotti L.A. (1939) Architetti e architetture militare. 3, gli architetti militari italiani nella Spagna, nel Portogallo e nelle loro colonie. Roma.

Marías F. (2001). "La memoria española de Francesco Paciotti: de Urbino al Escorial”. Anuario del Departamento de Historia y Teoría del Arte (UAM), vol. XIII, pp. 97-106.

Promis C. (1863). "La vita di Francesco Paciotto da Urbino”. Miscellanea di Storia italiana, IV.

Viganó M. (2004). El fratin mi ynginiero.

Sánchez Gijón A. (2000). "La Goleta, Bona, Bugía y África. Los presidios del reino de Túnez en la política mediterránea del Emperador”. En Las Fortificaciones de Carlos V. Madrid.

Vilar J.B. (1991). “Mapas, planos y fortificaciones hispánicos” en Túnez (S.XVI-XIX). Madrid. 